\title{
SINGULAR SETS OF HIGHER ORDER ELLIPTIC EQUATIONS
}

\author{
Qing Han, Robert Hardt, Fanghua Lin
}

\section{Introduction}

The implicit function theorem implies that the zero set of a smooth function, the set where the function vanishes, is a smooth hypersurface away from the critical zero set. Hence to study zero sets it is important to understand the structure of the critical zero sets. For solutions of the second order elliptic equations the critical zero sets represent the singular parts of zero sets. They have the Hausdorff dimension not greater than $n-2$. Hence sometimes they are called the singular sets of solutions. This result is not true for higher order elliptic equations. For example the critical zero sets may occupy the whole zero sets for some biharmonic polynomials. In order to study the zero sets of solutions of higher order elliptic equations we need to identify their singular parts and study their structure.

The singular sets of solutions to elliptic equations of the second order have been studied by many people. In [19], Hardt and Simon proved that for classical solutions with relatively high order derivatives singular sets are countable unions of subsets of correspondingly smooth $(n-2)$-dimensional submanifolds. Thus, they are countably $(n-2)$-rectifiable. See also [5]. This result was generalized to weak solutions in [14]. It is proved that for weak solutions, as long as they do not vanish to infinite order, the singular sets are countable unions of subsets of $C^{1, \alpha}(n-2)$-dimensional submanifolds, for some $\alpha \in(0,1)$. Hence they are also countably $(n-2)$-rectifiable. Concerning the size of singular sets, M. and T. Hoffmann-Ostenhof and N. Nadirashvili [20] showed that the singular sets of smooth solutions in three dimensional space have locally finite one dimensional Hausdorff measure. It was generalized to arbitrary dimensions, independently by one of the authors in [18] and M. and T. Hoffmann-Ostenhof and N. Nadirashvili

The authors are supported in part by NSF grants.

Typeset by $\mathcal{A M}_{\mathcal{S}}-\mathrm{T}_{\mathrm{EX}}$ 
[21]. In [16] we gave a uniform estimate on the measure of singular sets in terms of frequency of solutions.

In the present paper, we will study the singular sets of solutions to elliptic equations of arbitrary order. We will study solutions of finite differentiability in Euclidean spaces of arbitrary dimensions and give a uniform bound on the size of singular sets in terms of the highest vanishing order. Our method is similar to that in [16]. It avoids the complicated discussion of the complex dimension of the complex critical sets of real harmonic polynomials, needed in [18], [20] and [21].

Solutions to elliptic equations of the second order, having enough regularity, can not vanish to infinite order. This is the unique continuation property. However it is not true even for smooth solutions to equations of the higher order. Hence in order to study zero sets or singular sets we need to assume our solutions cannot vanish to infinite order.

Our main result is the following:

Main Theorem. Suppose that $u$ is a nonconstant solution of an equation

$$
\sum_{|\nu|=0}^{2 m} a_{\nu}(x) D^{\nu} u=0, \quad \text { in } B_{1}(0)
$$

where the coefficients $a_{\nu}$ are smooth in $B_{1}$ for any $|\nu| \leq 2 m$ and the leading coefficients satisfy the following assumption for some positive constant $\lambda$,

$$
\sum_{|\nu|=2 m} a_{\nu}(x) \xi^{\nu} \geq \lambda, \quad \forall \xi \in \mathbb{S}^{n-1} \subset \mathbb{R}^{n}, \quad x \in B_{1}(0)
$$

If $u$ does not vanish to infinite order in $B_{1}(0)$, then the singular set

$$
\mathcal{S}(u)=\left\{x \in B_{1} ; D^{\nu} u(x)=0, \text { for any }|\nu| \leq 2 m-1\right\}
$$

has locally finite $(n-2)$-dimensional Hausdorff measure, i.e., $\mathcal{H}^{n-2}\left(\mathcal{S}(u) \cap B_{r}\right)<$ $\infty$ for any $r \in(0,1)$.

In the following we will see that the local measure can be estimated uniformly in terms of the highest vanishing order and that the solution $u$ is not necessarily smooth. It is enough to assume that $u$ is differentiable with degree depending on the highest vanishing order. For the statement, see Theorem 3.2. 
The proof of the Main Theorem is based on two simple but important observations. First at almost all points in singular sets, the singular sets are approximated by $(n-2)$-dimensional hyperplanes and solutions are approximated by nonzero homogeneous polynomials of two variables by appropriate rotations. These polynomials satisfy some linear homogeneous elliptic equations with constant coefficients. Hence we need to focus only on those polynomials. The second obseravation is based on some simple algebra. In the two dimensional space any linear homogeneous elliptic operators of higher order with constant coefficients can be decomposed as the product of linear homogeneous elliptic operators of the second order with constants coefficients. This result is not true in the higher dimensional Euclidean spaces. Note that linear homogeneous elliptic operators of the second order with constants coefficients are essentially the Laplacian operator. Hence we are lead to the discussion of harmonic functions in the plane. An important tool in the whole discussion is the Weierstrass-Malgrange Preparation Theorem for finitely differential functions. We use this theorem to estimate the numbers of critical points of perturbations of harmonic functions in the plane.

We should emphasize that with the method in our paper we avoid the complicated discussion of all homogeneous polynomials satisfying constant coefficient elliptic equations of arbitrary order. It is not known that results in [18] or [21] for homogeneous harmonic polynomials are still true for those polynomials.

The paper is written as follows. In the first section, we discuss the geometric structure of singular sets. We prove a decomposition result which plays an important role in the proof of the Main Theorem. In section 2, we estimate the measure of singular sets away form the lower dimensional subsets. It is based on a perturbation argument. In section 3 we use the compactness argument to get the desired estimates on the geometric measure of singular sets. We do this for a certain class of operators and solutions which satisfy some nice compactness property.

\section{Geometric Structure of Singular Sets.}

In this section we discuss the geometric structure of singular sets.

Suppose that $L$ is a $2 m$-th order homogeneous elliptic linear operator in $B_{1}(0) \subset \mathbb{R}^{n}$ given by

$$
L \equiv \sum_{\substack{|\nu|=0 \\ 3}}^{2 m} a_{\nu}(x) D^{\nu}
$$


where the coefficients verify the following assumption for some positive constant $\lambda$ :

$$
\sum_{|\nu|=2 m} a_{\nu}(x) \xi^{\nu} \geq \lambda \quad \forall \xi \in \mathbb{S}^{n-1} \subset \mathbb{R}^{n}, \quad x \in B_{1}(0) .
$$

Suppose that $u$ is a nonconstant smooth solution of $L u=0$ in $B_{1}(0)$. We assume that $u$ does not vanish to infinite order in $B_{1}$. Then for any $p \in B_{1}$ there exists a homogeneous polynomial $P$ of degree $d$ such that

$$
\sum_{|\nu|=2 m} a_{\nu}(p) D^{\nu} P=0 \quad \text { in } \mathbb{R}^{n}
$$

and

$$
u(x+p)-P(x)=o\left(|x|^{d}\right) \quad \text { as } x \rightarrow 0 .
$$

We call $P$ the leading polynomial of $u$ at $p$. In fact the above estimate is also true for nonsmooth solutions. See [4] and [15].

We define the singular set $\mathcal{S}(u)$ as

$$
\mathcal{S}(u)=\left\{p \in B_{1} ; D^{\nu} u(p)=0 \quad \text { for any }|\nu| \leq 2 m-1\right\} .
$$

Theorem 1.1. Suppose that $L$ is an operator of the form (1.1) with smooth coefficients and satisfying (1.2). If $u$ satisfies $L u=0$ and does not vanish to infinite order in $B_{1}$, then $\mathcal{S}(u)$ is countably $(n-2)$-rectifiable. Moreover for $\mathcal{H}^{n-2}$ almost all points in $\mathcal{S}(u)$ the leading polynomials of the solution $u$ are functions of two variables by an appropriate rotation.

The proof is similar to that in [14]. For completeness we include most part of arguments with some improvement.

Proof. The proof consists of several steps.

Step 1. We first study the local behavior at each point.

For each integer $d \geq 2 m$, define the singular set of the $d$-th level

$$
\begin{gathered}
\mathcal{S}_{d}(u)=\left\{p \in B_{1} ;\right. \\
D^{i} u(p)=0, \quad \text { for any } i=0,1, \cdots, d-1, \\
\left.D^{d} u(p) \neq 0\right\} .
\end{gathered}
$$

Since $u$ does not vanish to infinite order, $\mathcal{S}_{d}(u)=\phi$ for sufficiently large $d$. Therefore

$$
\mathcal{S}(u)=\bigcup_{\substack{d \geq 2 m \\ 4}} \mathcal{S}_{d}(u)
$$


Take any point $y \in B_{1}(0) \cap \mathcal{S}_{d}(u)$. Suppose the leading polynomial of $u$ at $y$ is given by the $d$-degree non-zero homogeneous polynomial $P=P_{y}$. Then $P$ satisfies

$$
\sum_{|\nu|=2 m} a_{\nu}(y) D^{\nu} P=0 \quad \text { in } \mathbb{R}^{n}
$$

As for $u$ set

$$
\begin{aligned}
\mathcal{S}_{d}(P)=\left\{p \in B_{1} ;\right. & D^{i} P(p)=0, \quad \text { for any } i=0,1, \cdots, d-1, \\
& \left.D^{d} P(p) \neq 0\right\} .
\end{aligned}
$$

Since $P$ is a $d$-degree non-zero homogeneous polynomial, we have $0 \in \mathcal{S}_{d}(P)$.

We claim that $\mathcal{S}_{d}(P)$ is a linear subspace and

$$
P(x)=P(x+z) \quad \text { for any } \quad x \in \mathbb{R}^{n} \text { and } z \in \mathcal{S}_{d}(P) .
$$

In fact for any $z \in \mathcal{S}_{d}(P)$, we have

$$
D^{\nu} P(z)=0 \quad \text { for any }|\nu| \leq d-1 .
$$

Assume

$$
P(x)=\sum_{|\alpha|=d} a_{\alpha} x^{\alpha}
$$

Then we have

$$
P(x)=\sum_{|\alpha|=d} a_{\alpha}(x-z)^{\alpha} .
$$

This implies (1.4). Now we may prove that $\mathcal{S}_{d}(P)$ is a linear subspace easily.

Next, we prove that $\operatorname{dim} \mathcal{S}_{d}(P) \leq n-2$ for any $d \geq 2 m$. In fact the formula (1.4) implies $P$ is a function of $n$ - $\operatorname{dim} \mathcal{S}_{d}(P)$ variables. If $\operatorname{dim} \mathcal{S}_{d}(P)=n-1, P$ would be a $d$-degree monomial of one variable satisfying the equation (1.3). Hence $d<2 m$. This is a contradiction.

Step 2. We define for each $j=0,1,2, \ldots, n-2$,

$$
\mathcal{S}_{d}^{j}(u)=\left\{y \in \mathcal{S}_{d}(u) ; \operatorname{dim} \mathcal{S}_{d}\left(P_{y}\right)=j\right\} .
$$

We claim that $\mathcal{S}_{d}^{j}(u)$ is on a countable union of $j$-dimensional $C^{1}$ manifolds for any $d \geq 2 m$ and $j=0,1,2, \ldots, n-2$. In fact we will prove that for any $y \in \mathcal{S}_{d}^{j}$ 
there exists an $r=r(y)$ such that $\mathcal{S}_{d}^{j}(u) \cap B_{r}(y)$ is contained in a (single piece of) $j$-dimensional $C^{1}$ manifold.

To show this we let $\ell_{y}$ be the $j$-dimensional linear subspace $\mathcal{S}_{d}\left(P_{y}\right)$ for any $y \in \mathcal{S}_{d}^{j}(u)$. For any $\left\{y_{k}\right\} \subset \mathcal{S}_{d}^{j}(u)$ with $y_{k} \rightarrow y$, we first prove that

$$
\text { Angle }<\overline{y y_{k}}, \ell_{y}>\rightarrow 0
$$

To prove (1.5) we may assume $y=0$ and $\xi_{k}=y_{k} /\left|y_{k}\right| \rightarrow \xi \in \mathbb{S}_{1}$. Since $P$ is the leading polynomial of $u$ at $y=0$, there holds for $i=0,1, \cdots, d-1$,

$$
D^{i}(u(x)-P(x))=o\left(|x|^{d-i}\right) \quad \text { as } x \rightarrow 0
$$

Evaluating at $y_{k}=\left|y_{k}\right| \xi_{k}$ and taking the limit $k \rightarrow \infty$, we conclude that $D^{i} P(\xi)=0$ for any $i=0,1, \cdots, d-1$. Since $P_{y}$ is a $d$-degree homogeneous polynomial, then $\xi \in \ell_{y}=\mathcal{S}_{d}\left(P_{y}\right)$. This implies (1.5).

By (1.5) we obtain that for any $y \in \mathcal{S}_{d}^{j}(u)$ and small $\varepsilon>0$ there exists an $r=r(y, \varepsilon)$ such that

$$
\mathcal{S}_{d}^{j} \cap B_{r}(y) \subset B_{r}(y) \cap C_{\varepsilon}\left(\ell_{y}\right)
$$

or equivalently,

$$
\mathcal{S}_{d}^{j} \cap B_{r}(y) \cap\left(C_{\varepsilon}\left(\ell_{y}\right)\right)^{C}=\phi
$$

where

$$
C_{\varepsilon}\left(\ell_{y}\right)=\left\{z \in \mathbb{R}^{n} ; \operatorname{dist}\left(z, \ell_{y}\right) \leq \varepsilon|z|\right\} .
$$

Let $P_{k}$ and $P$ be the leading polynomials of $u$ at $y_{k}$ and $y=0$, respectively. By smoothness of the solution $u$ we have

$$
P_{k} \rightarrow P \quad \text { uniformly in } C^{d}\left(B_{1}(0)\right)
$$

This implies that

$$
\ell_{y_{k}} \rightarrow \ell_{y} \quad \text { as } k \rightarrow \infty
$$

as subspaces in $\mathbb{R}^{n}$. By an argument similar to above to prove (1.5) we may prove that the constant $r$ in (1.6) can be chosen uniformly for any point $z \in \mathcal{S}_{d}^{j}(u)$ in a neighborhood of $y$. In other words we obtain that for any $y \in \mathcal{S}_{d}^{j}(u)$ and any small $\varepsilon>0$ there exists an $r=r(\varepsilon, y)$ such that

$$
\mathcal{S}_{d}^{j}(u) \cap B_{r}(z) \subset B_{r}(z) \cap C_{\varepsilon}\left(\ell_{z}\right) \quad \text { for any } z \in \mathcal{S}_{d}^{j}(u) \cap B_{r}(y) \text {. }
$$


For $\varepsilon>0$ small enough this clearly implies that $\mathcal{S}_{d}^{j}(u) \cap B_{r}(y)$ is contained in a $j$-dimensional Lipschitz manifold. By (1.5) this manifold is $C^{1}$.

We may define

$$
\mathcal{S}^{j}(u)=\bigcup_{d \geq 2 m} \mathcal{S}_{d}^{j}(u) \quad \text { for } j=0,1, \cdots, n-2 .
$$

Then we have

$$
\mathcal{S}(u)=\bigcup_{j=0}^{n-2} \mathcal{S}^{j}(u)
$$

Moreover each $S^{j}(u)$ is on a countable union of $j$-dimensional $C^{1}$ manifolds for each $j=0,1, \cdots, n-2$. Now we set

$$
\begin{aligned}
\mathcal{S}_{\star}(u) & =\bigcup_{j=0}^{n-3} \mathcal{S}^{j}(u) \\
\mathcal{S}^{\star}(u) & =\mathcal{S}^{n-2}(u) .
\end{aligned}
$$

Then we have the desired decomposition

$$
\mathcal{S}(u)=\mathcal{S}^{\star}(u) \cup \mathcal{S}_{\star}(u)
$$

where $\mathcal{S}_{\star}(u)$ is countably $(n-3)$-rectifiable, $\mathcal{S}^{\star}(u)$ is on a countable union of $(n-2)$-dimensional $C^{1}$ manifolds and for any $y \in \mathcal{S}^{\star}(u)$ the leading polynomial of $u$ at $y$ is a homogeneous polynomial of 2 variables. (Q.E.D.)

Remark. It is clear from the proof that Theorem 1.1 still holds if $u$ is $C^{N}$ in $B_{1}$, with $N$ as the largest vanishing order of $u$ in $B_{1}$. The positive integer $N$ being the largest vanishing order of $u$ means for any $p \in B_{1}$ the leading polynomial of $u$ at $p$ is a homogeneous polynomial of degree not exceeding $N$.

\section{Geometric Measure of Good Parts in Singular Sets.}

Suppose that $L$ is a $2 m$-th order homogeneous elliptic linear operator in $B_{1}(0) \subset \mathbb{R}^{n}$ given by (1.1) with the property (1.2) and that $u$ is a nonconstant smooth solution of $L u=0$ in $B_{1}(0)$. We assume that $u$ does not vanish to infinite order in $B_{1}$ and let $N$ denote the largest vanishing order of $u$ in $B_{1}$. In other words for any $p \in B_{1}$ the leading polynomial of $u$ at $p$ is a homogeneous polynomial of degree not exceeding $N$. 
Theorem 2.1. Suppose that $L$ is an elliptic operator given by (1.1) with $C^{2 N^{2}}$ coefficients and (1.2) and that $u$ is a solution $L u=0$ in $B_{1}$, with $\|u\|_{L^{2}\left(B_{1}\right)}=$ 1 and $N$ as the largest vanishing order of $u$ in $B_{1}$. Then there exist positive constants $C(u)$ and $\varepsilon(u)$, depending on the solution $u$, and a finite collection of balls $\left\{B_{r_{i}}\left(x_{i}\right)\right\}$ with $r_{i} \leq 1 / 8$ and $x_{i} \in \mathcal{S}(u)$ such that for any $v \in C^{2 N^{2}+2 m-1}$ with

$$
|u-v|_{C^{2 N^{2}+2 m-1}\left(B_{1}\right)}<\varepsilon(u)
$$

there hold

$$
H^{n-2}\left(\mathcal{S}(v) \cap B_{1 / 2} \backslash \cup B_{r_{i}}\left(x_{i}\right)\right) \leq C(u)
$$

and

$$
\sum r_{i}^{n-2} \leq \frac{1}{2^{n-1}}
$$

where $C(u)$ also depends on $\lambda, n$ and $C^{2 N^{2}}$-norms of all coefficients of $L$.

Proof. Let $u$ be given as above. By Theorem 1.1 we have

$$
\mathcal{S}(u)=\mathcal{S}^{\star}(u) \cup \mathcal{S}_{\star}(u)
$$

where $\mathcal{S}_{\star}(u)$ has the Hausdorff dimension not exceeding $n-3, \mathcal{S}^{\star}(u)$ is on a countable union of $(n-2)$-dimensional $C^{1}$ manifolds and for any $p \in \mathcal{S}^{\star}(u)$ the leading polynomial of $u$ at $p$ is a homogeneous polynomial of 2 variables by an appropriate rotation. In particular

$$
H^{n-2}\left(\mathcal{S}_{\star}\left(u_{0}\right)\right)=0 .
$$

Then there exist at most countably many balls $B_{r_{i}}\left(x_{i}\right)$ with $r_{i} \leq 1 / 8$ and $x_{i} \in$ $\mathcal{S}_{\star}(u)$ such that

$$
\mathcal{S}_{\star}(u) \subset \bigcup_{i} B_{r_{i}}\left(x_{i}\right)
$$

and

$$
\sum r_{i}^{n-2} \leq \frac{1}{2^{n-1}}
$$

We claim for any $y \in \mathcal{S}^{\star}(u) \cap B_{3 / 4}$, there exist positive constants $R=$ $R(y, u)<1 / 8, r=r(y, u), \eta=\eta(y, u)$ and $c=c(y, u)$, with $r<R$, such that if the function $v$ satisfies

$$
|u-v|_{C^{2 N^{2}+2 m-1}\left(B_{R}(y)\right)}^{*}<\eta
$$


then

$$
H^{n-2}\left\{\mathcal{S}(v) \cap B_{r}(y)\right\} \leq c r^{n-2} .
$$

Here we use $\|\cdot\|_{C^{M}\left(B_{R}\right)}^{*}$ to denote, $M$ as a positive integer, the $C^{M}$-norm weighted with the radius $R$, i.e., for $w \in C^{M}\left(B_{R}\right)$,

$$
\|w\|_{C^{M}\left(B_{R}\right)}^{*}=\sum_{i=0}^{M} R^{i} \sup _{x \in B_{R}}\left|D^{i} w(x)\right|
$$

We will postpone the proof of (2.4).

It is obvious that the collection of $\left\{B_{r_{i}}\left(x_{i}\right)\right\}$ and $\left\{B_{r(y)}(y)\right\}, y \in \mathcal{S}^{\star}(u)$, covers $\mathcal{S}(u)$. By the compactness of $\mathcal{S}(u)$, there exist $x_{i} \in \mathcal{S}_{\star}(u), i=1, \cdots, k=k(u)$, and $y_{j} \in \mathcal{S}^{\star}(u), j=1, \cdots, l=l(u)$, such that

$$
\mathcal{S}(u) \cap B_{3 / 4} \subset\left(\bigcup_{i=1}^{k} B_{r_{i}}\left(x_{i}\right)\right) \bigcup\left(\bigcup_{j=1}^{l} B_{s_{j}}\left(y_{j}\right)\right)
$$

with $r_{i} \leq 1 / 8, i=1, \cdots, k$, and $s_{j} \leq 1 / 8, j=1, \cdots, l$. Since $\mathcal{S}(u)$ is closed, there exists a positive constant $\rho=\rho(u)$ such that

$$
\left\{x \in B_{3 / 4} ; \operatorname{dist}(x, \mathcal{S}(u))<\rho\right\} \subset\left(\bigcup_{i=1}^{k} B_{r_{i}}\left(x_{i}\right)\right) \bigcup\left(\bigcup_{j=1}^{l} B_{s_{j}}\left(y_{j}\right)\right) .
$$

It is easy to see that for such a $\rho$ there exists a positive constant $\delta=\delta(u)$ such that $|u-v|_{C^{2 m}\left(B_{3 / 4}\right)}<\delta$ implies

$$
\mathcal{S}(v) \cap B_{1 / 2} \subset\left\{x \in B_{3 / 4} ; \operatorname{dist}(x, \mathcal{S}(u))<\rho\right\} .
$$

Denote

$$
\mathcal{B}_{u}=\cup_{i=1}^{k} B_{r_{i}}\left(x_{i}\right), \quad \mathcal{G}_{u}=\cup_{j=1}^{l} B_{s_{j}}\left(y_{j}\right) .
$$

Now we take $\varepsilon(u)<\delta(u)$ small enough such that for any $v \in C^{2 N^{2}+2 m-1}$ in $B_{1}$ the condition

$$
|u-v|_{C^{2 N^{2}+2 m-1}\left(B_{1}\right)}<\varepsilon(u)
$$

implies that for each $j=1, \cdots, l=l(u)$,

$$
|u-v|_{C^{2 N^{2}+2 m-1}\left(B_{R}\left(y_{j}\right)\right)}^{*}<\eta\left(y_{j}, u\right) .
$$


Therefore there hold by $(2.1),(2.2),(2.5)-(2.7)$,

$$
\begin{gathered}
\mathcal{S}(v) \cap B_{1 / 2} \subset\left(\mathcal{S}(v) \cap \mathcal{B}_{u}\right) \cup\left(\mathcal{S}(v) \cap \mathcal{G}_{u}\right) \\
H^{n-2}\left(\mathcal{S}(v) \cap \mathcal{G}_{u}\right) \leq c \sum_{j=1}^{l} s_{j}^{n-2} \equiv C(u)
\end{gathered}
$$

and

$$
\mathcal{B}_{u}=\cup_{i=1}^{k} B_{r_{i}}\left(x_{i}\right), r_{i} \leq \frac{1}{8} \text { and } \sum_{i=1}^{k} r_{i}^{n-2} \leq \frac{1}{2^{n-1}}
$$

Now we prove (2.4) under the assumption (2.3).

For any $y \in \mathcal{S}^{\star}(u) \cap B_{3 / 4}$, there holds

$$
u(x+y)=P(x)+\psi(x)
$$

where $P$ is a nonzero $d$-degree homogeneous polynomial with $2 m \leq d \leq N$ and satisfies

$$
\sum_{|\nu|=2 m} a_{\nu}(y) D^{\nu} P=0 \quad \text { in } \mathbb{R}^{n}
$$

and $\psi(x)$ satisfies, by interior Schauder estimates, for some fixed $\alpha \in(0,1)$ and any $|x|<1 / 8$,

$$
\begin{aligned}
& \left|D^{i} \psi(x)\right| \leq C|x|^{d-i+\alpha} \quad \text { for } i=0,1, \cdots, d \\
& \left|D^{i} \psi(x)\right| \leq C \text { for } i=d+1, \cdots, 2 N^{2}+2 m-1
\end{aligned}
$$

where $C$ is a positive constant depending only on $N, \lambda, \alpha, n$ and $C^{2 N^{2}}$ norms of all coefficients $a_{\nu}$. By an appropriate rotation $P$ is a function of two variables. Hence we may assume $P$ is defined in $\mathbb{R}^{2} \times\{0\}$ with $\mathbb{R}^{n}=\mathbb{R}^{2} \times \mathbb{R}^{n-2}$. We abuse the notation by saying that $P$ is defined in $\mathbb{R}^{2}$. The operator

$$
L_{y} \equiv \sum_{\nu_{1}+\nu_{2}=2 m} a_{\left(\nu_{1}, \nu_{2}, 0, \cdots, 0\right)}(y) D_{x_{1}}^{\nu_{1}} D_{x_{2}}^{\nu_{2}}
$$

is a linear elliptic operator with constant coefficients and no lower order terms in $\mathbb{R}^{2}$. Elementary algebra asserts the following decomposition

$$
L_{y}=L_{1} \circ \cdots \circ L_{m}
$$


where each $L_{i}$ is a linear elliptic operator of the second order with constant coefficients and no lower order terms in $\mathbb{R}^{2}$. Hence

$$
L_{1} \circ \cdots \circ L_{m} P=0 \quad \text { in } \mathbb{R}^{2} \text {. }
$$

If $L_{2} \circ \cdots \circ L_{m} P$ is not identically zero, by letting $Q=L_{2} \circ \cdots \circ L_{m} P$ we get $L_{1} Q=0$. Otherwise

$$
L_{2} \circ \cdots \circ L_{m} P=0 \quad \text { in } \mathbb{R}^{2} \text {. }
$$

By repeating this process, we conclude that there exists a linear homogeneous differential operator $\mathcal{D}$ of order not exceeding $2 m-2$ such that $Q=\mathcal{D} P$ is a nonzero homogeneous polynomial satisfying

$$
L_{i} Q=0 \quad \text { in } \mathbb{R}^{2}
$$

for some $1 \leq i \leq m$. This implies $Q$ is a nonzero homogeneous harmonic polynomial by the change of coordinates if necessary. Hence we may apply Lemma 2.2 below to $Q$. Let $\varepsilon_{\star}$ and $r_{\star}$ be the constants given in Lemma 2.2 for $Q$. By (2.8) we may take a positive constant $R=R(y, u)<1 / 8$ such that

$$
\left\|\frac{1}{R^{d}} \psi\right\|_{C^{2 N^{2}+2 m-1}\left(B_{R}\right)}^{*}<\frac{1}{2} \varepsilon_{\star} .
$$

Choose $\eta$ small, depending on $R$ and $\varepsilon_{\star}$, such that (2.3) implies

$$
\left\|\frac{1}{R^{d}}(u-v)\right\|_{C^{2 N^{2}+2 m-1}\left(B_{R}(y)\right)}^{*}<\frac{1}{2} \varepsilon_{\star} .
$$

Then there holds

$$
\left\|\frac{1}{R^{d}}(v-P(\cdot-y))\right\|_{C^{2 N^{2}+2 m-1}\left(B_{R}(y)\right)}^{*}<\varepsilon_{\star} .
$$

By considering the transformation $x \mapsto y+R x$, we have

$$
\left\|\frac{1}{R^{d}} v(y+R \cdot)-P\right\|_{C^{2 N^{2}+2 m-1}\left(B_{1}\right)}<\varepsilon_{\star} .
$$

In particular there holds for the linear homogeneous differential operator $\mathcal{D}$ obtained above, with the order $l \leq 2 m-2$,

$$
\left\|\frac{1}{R^{d-l}} \mathcal{D} v(y+R \cdot)-Q\right\|_{C^{2 N^{2}+1}\left(B_{1}\right)}<\varepsilon_{\star} .
$$


Note $Q$ is a homogeneous harmonic polynomial of two variables and of degree $d-l \leq N$. Hence we may apply Lemma 2.2 to $Q$. After transforming back to $B_{R}(y)$ we get for some $r \leq R r_{\star}$

$$
\mathcal{H}^{n-2}\left(|D \mathcal{D} v|^{-1}\{0\} \cap B_{r}\right) \leq c(n)(d-1)^{2} r^{n-2} .
$$

Since $D \mathcal{D}$ is a differential operator of the order not exceeding $2 m-1$, hence $\mathcal{S}(v) \subset|D \mathcal{D} v|^{-1}\{0\}$. Therefore we obtain (2.4). (Q.E.D.)

The following result is used in the proof of Theorem 2.1. It was proved in [16]. We just point out some key steps.

Lemma 2.2. Let $P$ be a homogeneous harmonic polynomial of degree $d \geq 2$ and of two variables in $\mathbb{R}^{n}$. Then there exist positive constants $\varepsilon$ and $r$, depending on $P$, such that for any $u \in C^{2 d^{2}}\left(B_{1}\right)$ if

$$
|u-P|_{C^{2 d^{2}\left(B_{1}\right)}}<\varepsilon
$$

then

$$
\mathcal{H}^{n-2}\left(|D u|^{-1}\{0\} \cap B_{r}\right) \leq c(n)(d-1)^{2} r^{n-2} .
$$

The proof is based on the Weierstrass-Malgrange Preparation Theorem for finitely differentiable functions. First we recall some terminology. For any point $p \in \mathbb{R}^{n}$ we let $C_{p}^{\infty}\left(\mathbb{R}^{n}\right)$ denote the ring of germs of smooth functions in a neighborhood of $p$. For a smooth map $f$ from a neighborhood of $p$ into $\mathbb{R}^{n}$ with $f(p)=0$ we let $(f)$ denote the ideal generated by $f_{1}, \cdots, f_{n}$, the components of $f$. The local ring of $f$ at $p$ is the quotient ring

$$
\mathcal{R}_{f}(p)=C_{p}^{\infty}\left(\mathbb{R}^{n}\right) /(f) .
$$

It is easy to see that $\mathcal{R}_{f}(p)$ is a vector space over $\mathbb{R}$, whose dimension is called the multiplicity of $f$ at $p$. Instead of $C_{p}^{\infty}\left(\mathbb{R}^{n}\right)$ in the above definition we may also use $\mathbb{P}\left(\mathbb{R}^{n}\right)$, the space of all polynomials in $\mathbb{R}^{n}$, or $C_{p}^{\omega}\left(\mathbb{R}^{n}\right)$, the space of analytic germs at $p$. See $[A G V]$ or $[G G]$. The above notion can be defined for functions in $\mathbb{C}^{n}$. The importance of multiplicity is its connection with zeroes of maps. It can be shown that holomorphic maps, which maps zero to zero, have finite multiplicity at the origin if and only if the origin is the isolated zero point. This result is not true in $\mathbb{R}^{n}$, even for analytic maps.

The notion of local rings and multiplicities can also be defined for finitely differentiable functions.

The following result was proved in [2]. We assume that $\mu, N$ and $N^{\prime}$ are all positive integers with $N \leq N^{\prime}$. 
Lemma 2.3. Let $D \subset \mathbb{R}^{n}$ be a domain with $0 \in D$. Let $f: D \rightarrow \mathbb{R}^{n}$, with $f(0)=0$, be a function of smoothness $\mu\left(N^{\prime}+1\right)$ with the multiplicity $\mu$ at 0 , and let $\left\{e_{1}, \cdots, e_{\mu}\right\}$ be a basis of its local ring consisting of functions of smoothness $\mu(N+1)$. Then there exist neighborhoods $U, V$ and $Q$ of zero in $\mathbb{R}^{n}$, for which $V \subset U \subset D$ and $f(V) \subset Q \subset f(U)$, and a positive constant $\varepsilon$ with the following property: for any map $g: D \rightarrow \mathbb{R}^{n}$ of smoothness $\mu\left(N^{\prime}+1\right)$, if

$$
|f-g|_{C \mu\left(N^{\prime}+1\right)(D)}<\varepsilon
$$

there exists a bounded linear operator

$$
E^{g}=\left(E_{1}^{g}, \cdots, E_{\mu}^{g}\right): C^{\mu(N+1)}(U) \rightarrow\left[C^{N}(Q)\right]^{\mu},
$$

such that for any function $\varphi \in C^{N}(U)$ there holds

$$
\left.\varphi\right|_{V}=\sum_{i=1}^{\mu} e_{i} \cdot\left(E_{i}^{g} \varphi\right) \circ g .
$$

Proof of Lemma 2.2. We first prove for $n=2$. By using the polar coordinate $x_{1}=r \cos \theta$ and $x_{2}=r \sin \theta$ in $\mathbb{R}^{2}=\left\{\left(x_{1}, x_{2}\right)\right\}$ we may assume $P(x)=r^{d} \cos d \theta$. Direct calculation shows that

$$
D_{x_{1}} P=d r^{d-1} \cos (d-1) \theta, D_{x_{2}} P=-d r^{d-1} \sin (d-1) \theta .
$$

Therefore both $D_{x_{1}} P$ and $D_{x_{2}} P$ are products of $d-1$ different homogeneous linear functions. We obtain that the map $f=\left(D_{x_{1}} P, D_{x_{2}} P\right): \mathbb{R}^{2} \rightarrow \mathbb{R}^{2}$ has the origin as its only zero. In fact if $f$ is viewed as a map from $\mathbb{C}^{2}$ to $\mathbb{C}^{2}$, with $x \in \mathbb{R}^{2}$ replaced by $z \in \mathbb{C}^{2}$, the origin is also its only zero. Hence by Bezout's formula ([1], Corollary 1, P200) we conclude that

$$
\operatorname{dim} \mathbb{P}\left(\mathbb{R}^{2}\right) /(f) \leq(d-1)^{2},
$$

where $\mathbb{P}\left(\mathbb{R}^{2}\right)$ is the space of all polynomials in $\mathbb{R}^{2}$.

We may apply Lemma 2.3 with $N^{\prime}=N=1$ and $\mu=(d-1)^{2}$. We obtain that there exist neighborhoods $U, V, Q$ of the origin in $\mathbb{R}^{2}$ with $V \subset U \subset B_{1}$ and $f(V) \subset Q$ and a positive constant $\varepsilon>0$ such that for any map $g \in$ $C^{2(d-1)^{2}}\left(B_{1} ; \mathbb{R}^{2}\right)$ with $|g-f|_{C^{2(d-1)^{2}\left(B_{1}\right)}}<\varepsilon$ and any function $a \in C^{2(d-1)^{2}}(U)$ there exist $\alpha_{1}, \cdots, \alpha_{\mu} \in C^{1}(Q)$ such that

$$
a(x)=\sum_{i=1}^{\mu} e_{i}(x) \alpha_{i}(g(x)) \quad \text { for } x \in V .
$$


Hence for such a map $g$ we may prove for some positive constant $r$ with $B_{r} \subset V$

$$
\operatorname{Card}\left(g^{-1}\{0\} \cap B_{r}\right) \leq(d-1)^{2} .
$$

The proof is a modification of that for Lemma 2, P97, in [1].

Now consider $u \in C^{2 d^{2}}\left(B_{1}\right)$ with $|u-P|_{C^{2 d^{2}\left(B_{1}\right)}}<\varepsilon$. Note $2(d-1)^{2}+1 \leq 2 d^{2}$ for any positive integer $d$. Hence with $g=D u$ we have $|g-f|_{C^{2(d-1)^{2}\left(B_{1}\right)}}<\varepsilon$. Therefore we conclude

$$
\operatorname{Card}\left(|D u|^{-1}\{0\} \cap B_{r}\right) \leq(d-1)^{2}
$$

This finishes the proof for $n=2$.

Next we discuss the general dimension. For any $p \in \mathbb{R}^{n}$ and any $1 \leq i<j \leq n$ let $\mathbb{P}_{i j}(p)$ denote the 2-dimensional hyperplane

$$
\left\{\left(p_{1}, \cdots, p_{i-1}, x_{i}, p_{i+1}, \cdots, p_{j-1}, x_{j}, p_{j+1}, \cdots, p_{n}\right)\right\}
$$

and simply write $\mathbb{P}_{i j}(p)=\left\{\left(x_{i}, x_{j}\right)\right\}$ where there is no confusion. We also set $\mathbb{P}_{i j}=\mathbb{P}_{i j}(0)$.

Now let $P$ be a homogeneous harmonic polynomial of degree $d$ and of two variables in $\mathbb{R}^{n}$. With the explicit expression of $P$ we may find a change of coordinates with the following property. In the new coordinate system $\left\{\left(x_{1}, \cdots, x_{n}\right)\right\}$, for any fixed $1 \leq i<j \leq n$, the map $f_{i j}=\left(D_{x_{i}} P, D_{x_{j}} P\right) \mid \mathbb{P}_{i j}$, viewed as a map from $\mathbb{R}^{2}$ to $\mathbb{R}^{2}$, has the origin as its only zero and each component of $f_{i j}$ is the product of $d-1$ homogeneous linear polynomials. In fact if $f_{i j}$ is viewed as a map from $\mathbb{C}^{2}$ to $\mathbb{C}^{2}$, with $x \in \mathbb{R}^{n}$ replaced by $z \in \mathbb{C}^{n}$, the origin is also its only zero. As before there exist positive constants $\varepsilon_{i j}$ and $r_{i j}$ such that for any $g \in C^{2(d-1)^{2}}\left(B_{1 / 2}^{2} ; \mathbb{R}^{2}\right)$ with

$$
\left|g-f_{i j}\right|_{C^{2(d-1)^{2}}\left(B_{1 / 2}^{2}\right)}<\varepsilon_{i j}
$$

there holds

$$
\operatorname{card}\left(g^{-1}\{0\} \cap B_{r_{i j}}^{2}\right) \leq(d-1)^{2}
$$

Here we use $B_{r}^{2}$ to denote the ball (centered at origin) with radius $r$ in $\mathbb{R}^{2}$.

Take

$$
\varepsilon=\frac{1}{2} \min _{1 \leq i<j \leq n} \varepsilon_{i j}, \quad r=\min _{1 \leq i<j \leq n} r_{i j} .
$$


Consider any $u \in C^{2 d^{2}}\left(B_{1}\right)$ such that

$$
|u-P|_{C^{2 d^{2}\left(B_{1}\right)}}<\varepsilon
$$

For any $p \in \mathbb{R}^{n}$ and any $1 \leq i<j \leq n$, set $f_{i j}=\left(D_{x_{i}} P, D_{x_{j}} P\right) \mid \mathbb{P}_{i j}$ as before and $g_{i j, p}=\left(D_{x_{i}} u, D_{x_{j}} u\right) \mid \mathbb{P}_{i j}(p)$. We may take $r$ smaller such that for any $p \in B_{r}$ there holds

$$
\left|g_{i j, p}-f_{i j}\right|_{C^{2(d-1)^{2}}\left(B_{1 / 2}^{2}\right)}<2 \varepsilon \leq \varepsilon_{i j}
$$

Hence

$$
\operatorname{card}\left(g_{i j, p}^{-1}\{0\} \cap B_{r}^{2}\right) \leq(d-1)^{2} .
$$

Obviously $|D u|^{-1}\{0\} \cap \mathbb{P}_{i j}(p) \subset g_{i j, p}^{-1}\{0\}$. If we set $\pi_{i j}$ as the projection

$$
\pi_{i j}\left(x_{1}, \cdots, x_{n}\right)=\left(x_{1}, \cdots, x_{i-1}, x_{i+1}, \cdots, x_{j-1}, x_{j+1}, \cdots, x_{n}\right) \in \mathbb{R}^{n-2}
$$

then we have shown that for any $q \in B_{r}^{n-2} \subset \mathbb{R}^{n-2}$ and any $1 \leq i<j \leq n$

$$
\operatorname{card}\left(|D u|^{-1}\{0\} \cap \pi_{i j}^{-1}(q) \cap B_{r}\right) \leq(d-1)^{2} .
$$

Hence the integral geometric formula [10], 3.2.22, implies

$$
\begin{aligned}
& \mathcal{H}^{n-2}\left(|D u|^{-1}\{0\} \cap B_{r}\right) \\
\leq & \sum_{1 \leq i<j \leq n} \int_{B_{r}^{n-2}} \operatorname{card}\left(|D u|^{-1}\{0\} \cap \pi_{i j}^{-1}(q) \cap B_{r}\right) d \mathcal{H}^{n-2} q \\
\leq & c(n)(d-2)^{2} r^{n-2} .
\end{aligned}
$$

(Q.E.D.)

\section{Compact classes of operators and solutions.}

The proof of the Main Theorem is based on an iteration of Theorem 2.1. In order to do this we need to introduce a class of elliptic operators which is invariant under translation and scaling. For some constants $\lambda, \alpha \in(0,1), K>0$ and some nonnegative integer $M$, we define a class of linear elliptic operator of order $2 m, \mathcal{L}(\lambda, M, \alpha, K)$, as follows. Let

$$
L \equiv \sum_{|\nu|=0}^{2 m} a_{\nu}(x) D^{\nu}
$$


be an elliptic operator of order $2 m$ defined on $B_{1}(0) \subset \mathbb{R}^{n}$. We say $L \in$ $\mathcal{L}(\lambda, M, \alpha, K)$ if those coefficients $a_{\nu},|\nu| \leq 2 m$, satisfy the following conditions:

$$
\sum_{|\nu|=2 m} a_{\nu}(x) \xi^{\nu} \geq \lambda, \quad \forall \xi \in \mathbb{S}^{n-1} \subset \mathbb{R}^{n}, \quad x \in B_{1}(0)
$$

and

$$
\sum_{|\nu|=0}^{2 m}\left\|a_{\nu}\right\|_{C^{M, \alpha}\left(B_{1}\right)} \leq K .
$$

We note that if $L \in \mathcal{L}(\lambda, M, \alpha, K)$ and $u \in W^{2 m, 2}\left(B_{1}\right)$ satisfy $L u=0$ in $B_{1}$, then by the interior Schauder estimates there holds for any $r \in(0,1)$,

$$
\|u\|_{C^{M+2 m, \alpha}\left(B_{1-r}\right)} \leq C(r)\|u\|_{L^{2}\left(B_{1}\right)},
$$

where $C(r)$ is a positive constant which also depends on $\lambda, M, \alpha, K$ and the dimension $n$.

Let $L \in \mathcal{L}(\lambda, M, \alpha, K)$ and $x_{0} \in B_{1}, 0<\rho \leq \operatorname{dist}\left(x_{0}, \partial B_{1}\right)$, then the operator $L_{x_{0}, \rho}$ defined by

$$
L_{x_{0}, \rho} \equiv \sum_{|\nu|=0}^{2 m} \rho^{2 m-|\nu|} a_{\nu}\left(x_{0}+\rho x\right) D^{\nu}
$$

belongs to $\mathcal{L}(\lambda, M, \alpha, K)$. This translation and scaling invariant property of $\mathcal{L}(\lambda, M, \alpha, K)$ turns out to be very useful.

Finially in order to control the vanishing order quantitatively we introduec the doubling condition. Consider a positive integer $N$. A function $u \in L^{2}\left(B_{1}\right)$ is said to belong to $\mathcal{S}_{N}$ if

$$
f_{B_{2 r}\left(x_{0}\right)} u^{2}(x) d x \leq 4^{N} f_{B_{r}\left(x_{0}\right)} u^{2}(x) d x,
$$

for all $x_{0} \in B_{2 / 3}$ and $0<2 r<\operatorname{dist}\left(x_{0}, \partial B_{1}\right)$. It is easy to check that nonzero functions satisfying the doubling condition cannot vanish to infinite order. In fact for $u \in C^{N}\left(B_{1}\right)$ satisfying (3.4) the leading polynomial of $u$ at any point $x_{0} \in B_{2 / 3}$ has the degree not exceeding $N$. The converse is also true, namely, functions satisfy the doubling condition if they do not vanish to infinite order. In this case the constant $N$ in the doubling condition is much larger than the largest vanishing order.

We now define $\mathcal{S}_{N}(\lambda, M, \alpha, K)$ as the collection of all functions $u$ in $\mathcal{S}_{N}$ and satifying $L u=0$ in $B_{1}$ for some $L \in \mathcal{L}(\lambda, M, \alpha, K)$.

The class $\mathcal{S}_{N}(\lambda, M, \alpha, K)$ has the following important compactness property. 
Lemma 3.1. For any fixed positive constants $\lambda, \alpha<1$ and $K$ and nonnegative integers $N$ and $M$, the collection

$$
\left\{u \in \mathcal{S}_{N}(\lambda, M, \alpha, K) ; \int_{B_{1 / 2}} u^{2}(x) d x=1\right\}
$$

is compact under the local $L^{\infty}$-metric.

Proof. The proof is straightforward. Suppose $u_{k} \in \mathcal{S}_{N}$ and $L_{k} \in \mathcal{L}(\lambda, M, \alpha, K)$ satisfy $L_{k} u_{k}=0$ in $B_{1}$ with $\int_{B_{1 / 2}} u_{k}^{2}(x) d x=1$. By (3.4) and some covering argument there holds for any $R \in(0,1)$

$$
\left\|u_{k}\right\|_{L^{2}\left(B_{R}\right)} \leq c(N, R), \quad k=1,2, \cdots
$$

Interior Schauder estimates imply

$$
\left\|u_{k}\right\|_{C^{M+2 m, \alpha}\left(B_{R}\right)} \leq c(N, R), \quad k=1,2, \cdots
$$

Then there is a subsequence $u_{k^{\prime}}$ such that $u_{k^{\prime}}$ converges to $u$ in $C_{l o c}^{M+2 m}\left(B_{1}\right)$ with $L u=0$ for some $L \in \mathcal{L}(\lambda, M, \alpha, K)$. In (3.4) with $u$ replaced with $u_{k}$, we may take the limit $k \rightarrow \infty$. Hence (3.4) holds for $u$ and then $u \in \mathcal{S}_{N}$. It is obvious that $\int_{B_{1 / 2}} u^{2}(x) d x=1$. (Q.E.D.)

Now we prove the following result.

Theorem 3.2. Let $\lambda, \alpha$ and $K$ be positive constants with $\lambda, \alpha<1$ and $N$ a positive integer. Then there holds for any $u \in \mathcal{S}_{N}\left(\lambda, 2 N^{2}, \alpha, K\right)$

$$
H^{n-2}\left\{\mathcal{S}(u) \cap B_{1 / 2}\right\} \leq C
$$

where $C$ is a positive constant depending on $N$, as well as $\lambda, \alpha, K$ and $n$.

The Main Theorem follows readily from Theorem 3.2.

To prove Theorem 3.2 we need an improved version of Theorem 2.1.

Lemma 3.3. Suppose $N, \lambda, \alpha$ and $K$ are given positive constants with $\lambda, \alpha<1$. Then there exists a positive constant $C$, depending on $N$, as well as $\lambda, \alpha, K$ and $n$, such that for any $u \in \mathcal{S}_{N}\left(\lambda, 2 N^{2}, \alpha, K\right)$ there exists a finite collection of balls $\left\{B_{r_{i}}\left(x_{i}\right)\right\}$, with $r_{i} \leq 1 / 4$ and $x_{i} \in \mathcal{S}(u)$, such that

$$
H^{n-2}\left(\mathcal{S}(u) \cap \underset{17}{\left.B_{1 / 2} \backslash \cup B_{r_{i}}\left(x_{i}\right)\right) \leq C}\right.
$$


and

$$
\sum r_{i}^{n-2} \leq \frac{1}{2}
$$

Proof. With $M=2 N^{2}$, we set

$$
\mathcal{S}_{N}^{1}=\mathcal{S}_{N}^{1}(\lambda, M, \alpha, K)=\left\{u \in \mathcal{S}_{N}(\lambda, M, \alpha, K) ; \int_{B_{1 / 2}} u^{2}=1\right\} .
$$

Take an arbitrary solution $u_{0} \in \mathcal{S}_{N}^{1}$. For any $u \in \mathcal{S}_{N}^{1}$, the condition $\mid u_{0}-$ $\left.u\right|_{L^{\infty}\left(B_{7 / 8}\right)}<\eta_{0}$ implies by interior Schauder estimates

$$
\left|u_{0}-u\right|_{C^{M+2 m}\left(B_{3 / 4}\right)} \leq c\left(\eta_{0}\right)
$$

where $c\left(\eta_{0}\right) \rightarrow 0$ as $\eta_{0} \rightarrow 0$. We take $\eta_{0}=\eta_{0}\left(u_{0}\right)$ small such that

$$
c\left(\eta_{0}\right) \leq \varepsilon\left(u_{0}\right)
$$

where $\varepsilon\left(u_{0}\right)$ is the constant given in Theorem 2.1. Then by Theorem 2.1 there exist a positive constant $C\left(u_{0}\right)$ and finitely many balls $\left\{B_{r_{i}}\left(x_{i}\right)\right\}$, with $x_{i} \in \mathcal{S}\left(u_{0}\right)$ and $r_{i} \leq 1 / 8$, such that for any $u \in S_{N}^{1}$ with $\left|u_{0}-u\right|_{L^{\infty}\left(B_{7 / 8}\right)}<\eta_{0}$, there hold

$$
H^{n-2}\left(\mathcal{S}(u) \cap B_{\frac{1}{2}} \backslash \cup_{i \geq 1} B_{r_{i}}\left(x_{i}\right)\right) \leq C\left(u_{0}\right)
$$

and

$$
\sum_{i \geq 1} r_{i}^{n-2} \leq \frac{1}{2^{n-1}}
$$

If $\mathcal{S}(u) \cap B_{r_{i}}\left(x_{i}\right) \neq \phi$, we may take $\tilde{x}_{i} \in \mathcal{S}(u) \cap B_{r_{i}}\left(x_{i}\right)$. Obviously $B_{r_{i}}\left(x_{i}\right) \subset$ $B_{2 r_{i}}\left(\tilde{x}_{i}\right)$. Therefore for such a $u$ by renaming radii and centers we find a finite collection of balls $\left\{B_{r_{i}}\left(x_{i}\right)\right\}$, with $x_{i} \in \mathcal{S}(u)$ and $r_{i} \leq 1 / 4$, such that

$$
H^{n-2}\left(\mathcal{S}(u) \cap B_{1 / 2} \backslash \cup B_{r_{i}}\left(x_{i}\right)\right) \leq C\left(u_{0}\right)
$$

and

$$
\sum_{i \geq 1} r_{i}^{n-2} \leq \frac{1}{2}
$$

By Lemma 3.1, $\mathcal{S}_{N}^{1}$ is compact under local $L^{\infty}$-metric. Hence there exist $u_{1}, \cdots$, $u_{p} \in \mathcal{S}_{N}^{1}$ and $\eta_{1}=\eta\left(u_{1}\right), \cdots, \eta_{p}=\eta\left(u_{p}\right)$ such that for any $u \in \mathcal{S}_{N}^{1}$ there exists a $k$ with $1 \leq k \leq p$ with the property

$$
\left|u-u_{k}\right|_{L^{\infty}\left(B_{7 / 8}\right)} \leq \eta_{k}
$$


Denote

$$
C=\max \left\{C\left(u_{1}\right), \cdots, C\left(u_{p}\right)\right\} .
$$

Such a constant $C$ is finite and depends on the class $\mathcal{S}_{N}\left(\lambda, 2 N^{2}, \alpha, K\right)$. This finishes the proof. (Q.E.D.)

Proof of Theorem 3.2. We use the standard iteration process to prove Theorem 3.2. To begin with, define

$$
\phi_{0}=\left\{B_{1 / 2}(0)\right\}
$$

We claim that we may find $\phi_{1}, \phi_{2}, \cdots$, each of which consists of a collection of balls, such that for any $\ell \geq 1$

$$
\begin{gathered}
\operatorname{rad}(B) \leq \frac{1}{2} \cdot\left(\frac{1}{2}\right)^{\ell} \quad \text { for any } B \in \phi_{\ell} \\
\sum_{B \in \phi_{\ell}}[\operatorname{rad}(B)]^{n-2} \leq\left(\frac{1}{2}\right)^{\ell}
\end{gathered}
$$

and

$$
H^{n-2}\left(\mathcal{S}(u) \cap \bigcup_{B \in \phi_{\ell-1}} B \sim \bigcup_{B \in \phi_{\ell}} B\right) \leq C\left(\frac{1}{2}\right)^{\ell-1}
$$

where $C$ is the positive constant given in Lemma 3.3. Observe that

$$
\begin{aligned}
\mathcal{S}(u) \cap B_{1 / 2}(0) \subset \bigcup_{\ell=1}^{\infty}\left(\mathcal{S}(u) \cap\left(\bigcup_{B \in \phi_{\ell-1}} B \sim \bigcup_{B \in \phi_{\ell}} B\right)\right) \\
\\
\cup \bigcap_{\ell=0}^{\infty}\left(\mathcal{S}(u) \cap \bigcup_{j=\ell}^{\infty} \bigcup_{B \in \phi_{j}} B\right) .
\end{aligned}
$$

Hence we have

$$
H^{n-2}\left(\mathcal{S}(u) \cap B_{1 / 2}(0)\right) \leq C\left\{\sum_{\ell \geq 1}\left(\frac{1}{2}\right)^{\ell-1}+\inf _{\ell \geq 1} \sum_{j=\ell}^{\infty}\left(\frac{1}{2}\right)^{j}\right\} \leq 2 C .
$$

To prove the claim we construct $\left\{\phi_{\ell}\right\}$ by induction. Note $\phi_{0}=\left\{B_{1 / 2}\right\}$. Suppose $\phi_{0}, \phi_{1}, \ldots, \phi_{\ell-1}$ are already defined for some $\ell \geq 1$. To construct $\phi_{\ell}$ we 
take $B=B_{r}(y) \in \phi_{\ell-1}$, with $r \leq 1 / 2$. Consider the transformation $x \mapsto y+2 r x$. Then, via $L u=0$ in $B_{2 r}(y)$, we have

$$
\widetilde{L} \tilde{u}=0 \quad \text { in } \quad B_{1}(0),
$$

where

$$
\widetilde{L}=\sum_{|\nu|=0}^{2 m}(2 r)^{2 m-|\nu|} a_{\nu}(y+2 r x) D_{x}^{\nu}
$$

and

$$
\tilde{u}(x)=u(y+2 r x) .
$$

By the discussion in the beginning of the present section we get $\tilde{u} \in \mathcal{S}_{N}(\lambda, M, \alpha, K)$. Hence we may apply Lemma 3.3 to $\tilde{u}$ to obtain a collection of balls $\left\{B_{s_{i}}\left(z_{i}\right)\right\}$, with $s_{i} \leq 1 / 4$ and $z_{i} \in \mathcal{S}(\tilde{u})$ such that

$$
H^{n-2}\left(\mathcal{S}(\tilde{u}) \cap B_{1 / 2} \backslash \cup B_{s_{i}}\left(z_{i}\right)\right) \leq C
$$

and

$$
\sum s_{i}^{n-2} \leq \frac{1}{2} .
$$

Now transform $B_{1 / 2}(0)$ back to $B_{r}(y)$ by $x \mapsto(x-y) / 2 r$. We obtain that for $B=B_{r}(y) \in \phi_{\ell-1}$, there exist finitely many balls $\left\{B_{r_{i}}\left(x_{i}\right)\right\}$ in $B_{2 r}(y)$, with $r_{i} \leq 1 / 2$, such that

$$
H^{n-2}\left(\mathcal{S}(u) \cap B_{r}(y) \backslash \bigcup_{i} B_{r_{i}}\left(x_{i}\right)\right) \leq C r^{n-2}
$$

and

$$
\sum_{i} r_{i}^{n-2} \leq \frac{1}{2} r^{n-2}
$$

Then we set

$$
\phi_{\ell}^{B}=\bigcup_{i}\left\{B_{i}\left(x_{i}\right)\right\}
$$

and

$$
\phi_{\ell}=\bigcup_{\substack{B \in \phi_{\ell-1} \\ 20}} \phi_{\ell}^{B}
$$


Hence we obtain

$$
H^{n-2}\left(\mathcal{S}(u) \cap \bigcup_{B \in \phi_{\ell-1}} B \sim \bigcup_{B \in \phi_{\ell}} B\right) \leq C\left(\sum_{B_{r_{i}}\left(x_{i}\right) \in \phi_{\ell-1}} r_{i}^{n-2}\right)
$$

and by induction

$$
r_{i} \leq \frac{1}{2} \cdot\left(\frac{1}{2}\right)^{\ell}, \quad \sum_{B_{r_{i}}\left(x_{i}\right) \in \phi_{\ell}} r_{i}^{n-2} \leq\left(\frac{1}{2}\right)^{\ell}
$$

for each $\ell \geq 1$. This concludes the proof. (Q.E.D.)

\section{REFERENCES}

[1] V.I. Arnold, S.M. Gusein-Zade, and A.N. Varchenko, Singularities of Differentiable Maps, Volume I, Birkhauser, 1985.

[2] V.I. Bakhtin, Weierstrass-Malgrange preparation theorem for finitely smooth case, Functional Anal. Appl., 24, 1990, 86-96.

[3] V.I. Bakhtin, Weierstrass preparation theorem for finitely smooth modules, Theory of Singularities and its Applications, Advances in Soviet Mathematics, Volume 1, 287-294.

[4] L. Bers, Local behavior of solution of general linear elliptic equations, Comm. Pure Appl. Math. 8, 1955, 473-496.

[5] L.A. Caffarelli, and A.Friedman, Partial regularity of the zero-set of solutions of linear and superlinear elliptic equations, J. Diff. Eq. 60, 1985, 420-433.

[6] J.P. D'Angelo, Several Complex Variables and the Geometry of Real Hypersurfaces, CRC Press, Inc., 1992.

[7] R.-T. Dong, Nodal sets of eigenfunctions on Riemann surfaces, J. Diff. Geom. 36, 1992, 493-506.

[8] H. Donnelly, and C. Fefferman, Nodal sets of eigenfunctions on Riemannian manifolds, Invent. Math. 161-183.

[9] H. Donnelly, and C. Fefferman, Nodal sets for eigenfunctions of the Laplacian on surfaces, J. Amer. math. Soc. 3, 1990, 333-353.

[10] H. Federer, Geometric Measure Theory, Springer-Verlag, New York, 1969.

[11] N. Garofalo, and F.-H. Lin, Monotonicity properties of variational integrals, $A_{p}$ weights and unique continuation, Indiana Univ. Math J. 35, 1986, 245-267.

[12] D. Gilbarg and N. Trudinger, Elliptic Partial Differential Equations of Second Order, Second Edition, Springer, Berlin, 1983.

[13] M. Golubitsky and V. Guillemin, Stable Mappings and Their Singularities, SpringerVerlag, New York, 1973.

[14] Q. Han, Singular sets of solutions to elliptic equations, Indiana Univ. Math J. 43, 1994, 983-1002.

[15] Q. Han, Pointwise Schauder estimates with applications to nodal sets, to appear. 
[16] Q. Han, R. Hardt and F.-H. Lin, Geometric measure of singular sets of elliptic equations, preprint.

[17] Q. Han and F.-H. Lin, On the geometric measure of nodal sets of solutions, J Partial Diff. Eq., 7, 1994, 111-131.

[18] R. Hardt, Critical set of an elliptic equation, preprint.

[19] R. Hardt, and L. Simon, Nodal sets for solutions of elliptic equations, J. Diff. Geom. 30, 1989, 505-522.

[20] M. Hoffman-Ostenhof, T. Hoffman-Ostenhof and N. Nadirashvili, Critical sets of smooth solutions to elliptic equations in dimension 3, Indiana Univ. Math J. 45, 1996, 15-37.

[21] M. Hoffman-Ostenhof, T. Hoffman-Ostenhof and N. Nadirashvili, Critical sets of smooth solutions to elliptic equations, preprint.

[22] F.-H. Lin, Nodal sets of solutions of elliptic and parabolic equations, Comm. Pure Appl. Math. 45, 1991, 287-308.

[23] B. Malgrange, Ideals of Differentiable Mappings , Oxford Univ. Press, Oxford, 1966.

[24] R. Narasimhan, Introduction to the Theory of Analytic Spaces, Lecture Notes in Mathematics, No. 25, Springer-Verlag, Berlin-New York, 19661966.

[25] L. Simon, Lectures on Geometric Measure Theory, Proc. C.M.A., Aust. Nat. Univ. 1983.

[26] S.T. Yau, Open problems in geometry, Proc. Symp. Pure Math., 54, 1993, 1-28.

[27] Y. Yomdin, The set of zeroes of an almost polynomial functions, , Proc. Amer. Math. Soc., 90(4), 1984, 538-542.

Department of Mathematics, University of Notre Dame, Notre Dame, IN 46556 E-mail address: qhan@yansu.math.nd.edu

Department of Mathematics, Rice University, Houston, TX 77251

E-mail address: hardt@math.rice.edu

Courant Institute, 251 Mercer Street, New York, NY 10012

E-mail address: linf@math1.cims.nyu.edu 